\title{
A Note on Various Classes of Compatible-Type Pairs of Mappings and Common Fixed Point Theorems
}

\author{
Zoran Kadelburg, ${ }^{1}$ Stojan Radenović, ${ }^{2}$ and Naseer Shahzad ${ }^{3}$ \\ ${ }^{1}$ Faculty of Mathematics, University of Belgrade, Studentski trg 16, 11000 Beograd, Serbia \\ ${ }^{2}$ Faculty of Mechanical Engineering, University of Belgrade, Kraljice Marije 16, 11120 Beograd, Serbia \\ ${ }^{3}$ Department of Mathematics, King Abdulaziz University, P.O. Box 80203, Jeddah 21589, Saudi Arabia
}

Correspondence should be addressed to Naseer Shahzad; nshahzad@kau.edu.sa

Received 8 September 2013; Accepted 27 September 2013

Academic Editor: Wei-Shih Du

Copyright (C) 2013 Zoran Kadelburg et al. This is an open access article distributed under the Creative Commons Attribution License, which permits unrestricted use, distribution, and reproduction in any medium, provided the original work is properly cited.

Several authors have introduced various conditions which can be used in order to prove common fixed point results. However, it became clear recently that some of these conditions, though formally distinct from each other, actually coincide in the case when the given mappings have a unique point of coincidence. Hence, in fact, new common fixed point results cannot be obtained in this way. We make a review of such connections and results in this paper.

\section{Introduction}

The simplest common fixed point results for mappings $A, S$ : $X \rightarrow X$ can be obtained if $A$ and $S$ commute (Jungck, [1]). Obviously, this condition is too strong, and so it is natural to seek for weaker assumptions. Hence, several authors have introduced various other conditions (we will call them compatible-type conditions) which can be used in order to prove common fixed point results. Some of these conditions were given in [2-19]. These (and other) conditions were used in other papers cited in the references. A review of the relationship between various compatible-type conditions introduced until 2001 was given in [20].

However, it became clear recently that some of these conditions, though formally distinct from each other, actually coincide in the case when the given mappings have a unique point of coincidence. Hence, in fact, new common fixed point results cannot be obtained in this way. We make a review of such connections and results in this paper.

\section{Definitions and Relations between Various Types of Pairs}

Most of the notions and results that follow can be formulated and proved in various types of spaces-metric, symmetric, cone metric, $b$-metric, probabilistic metric, and so forth. For the sake of simplicity, we will stay within the framework of metric spaces.

Let $(X, d)$ be a metric space, and let $A, S: X \rightarrow X$. We will denote by $C(A, S)$ the set of coincidence points (CP) of $A$ and $S$, that is,

$$
C(A, S)=\{x \in X \mid A x=S x\},
$$

by $P C(A, S)$ the set of points of coincidence (POC) of $A$ and $S$, that is,

$$
P C(A, S)=\{y \in X \mid y=A x=S x \text {, for some } x \in X\},
$$

and by $\mathscr{L}(A, S)$ the set of sequences $\left(x_{n}\right)$ in $X$ satisfying $\lim _{n \rightarrow \infty} A x_{n}=\lim _{n \rightarrow \infty} S x_{n}$, that is,

$$
\mathscr{L}(A, S)=\left\{\left(x_{n}\right) \mid \lim _{n \rightarrow \infty} A x_{n}=\lim _{n \rightarrow \infty} S x_{n}\right\} .
$$

The following are definitions of some of the multitude of compatible-type conditions, introduced and used for establishing common fixed point results in recent decades. 
TABLE 1

\begin{tabular}{lcc}
\hline If & Properties that trivially hold & Properties that cannot hold \\
\hline$C(A, S)=\emptyset$ & $(7),(9),(19)$ & $(8),(14),(15),(16),(17),(20)$ \\
\hline $\mathscr{L}(A, S)=\emptyset$ & $(3),(6),(11),(13),(18)$ & $(2),(4),(5),(12)$ \\
& $(7),(9),(10),(19)$ & $(8),(14),(15),(16),(17),(20)$ \\
\hline & $(7) \Rightarrow(8),(8) \Leftrightarrow(9),(10) \Rightarrow(8)$ \\
& $(8) \Rightarrow(17),(19) \Rightarrow(20)$ & \\
& $(2),(3) \Rightarrow(6),(5) \Leftrightarrow(6)$ & \\
& $(11) \Rightarrow(12),(12) \Leftrightarrow(13)$ & \\
$\mathscr{L}(A, S) \neq \emptyset$ & $(2),(3) \Rightarrow(6),(5) \Leftrightarrow(6)$ & \\
& $(11) \Rightarrow(12),(12) \Leftrightarrow(13)$ & \\
\hline
\end{tabular}

Definition 1. It is said that the pair $(A, S)$ is

(1) weakly commuting [2] if, for all $x \in X, d(A S x, S A x) \leq$ $d(A x, S x)$

(2) said to satisfy the property (E.A) [7] if $\mathscr{L}(A, S) \neq \emptyset$ (i.e., if there exists a sequence $\left(x_{n}\right)$ in $X$ such that $\left.\lim _{n \rightarrow \infty} A x_{n}=\lim _{n \rightarrow \infty} S x_{n}\right)$

(3) compatible [3] if, for all $\left(x_{n}\right),\left(x_{n}\right) \in \mathscr{L}(A, S)$ implies that $\lim _{n \rightarrow \infty} d\left(A S x_{n}, S A x_{n}\right)=0$;

(4) noncompatible [4] if, for some $\left(x_{n}\right) \in \mathscr{L}(A, S)$, $\lim _{n \rightarrow \infty} d\left(A S x_{n}, S A x_{n}\right) \neq 0$ or does not exist;

(5) subcompatible [15] if, for some $\left(x_{n}\right) \in \mathscr{L}(A, S)$, $\lim _{n \rightarrow \infty} d\left(A S x_{n}, S A x_{n}\right)=0$

(6) conditionally compatible [6] if $\mathscr{L}(A, S) \neq \emptyset$ implies that for some $\left(x_{n}\right) \in \mathscr{L}(A, S), \lim _{n \rightarrow \infty} d\left(A S x_{n}\right.$, $\left.S A x_{n}\right)=0$;

(7) weakly compatible [5] if, for all $x \in X, A x=S x$ implies that $A S x=S A x$ (i.e., if, for all $x \in X, x \in$ $C(A, S)$ implies that $A x \in C(A, S)$;

(8) occasionally weakly compatible [8] (see also [21-23]) if, for some $x \in X, A x=S x$ and $A S x=S A x$ (i.e., if, for some $x \in C(A, S), A x \in C(A, S)$ );

(9) conditionally commuting [9] if $C(A, S) \neq \emptyset$ implies that there exists $\emptyset \neq Y \subseteq C(A, S)$ such that, for all $y \in Y, A S y=S A y$;

(10) faintly compatible [16] if it is conditionally compatible and conditionally commuting (i.e., (6) and (9) hold);

(11) reciprocally continuous [13] if, for all $\left(x_{n}\right) \in \mathscr{L}(A, S)$, $\lim _{n \rightarrow \infty} A x_{n}=\lim _{n \rightarrow \infty} S x_{n}=z$ implies that $\lim _{n \rightarrow \infty} A S x_{n}=A z$ and $\lim _{n \rightarrow \infty} S A x_{n}=S z$;

(12) subsequentially continuous [15] if, for some $\left(x_{n}\right) \in$ $\mathscr{L}(A, S), \lim _{n \rightarrow \infty} A x_{n}=\lim _{n \rightarrow \infty} S x_{n}=z$ and $\lim _{n \rightarrow \infty} A S x_{n}=A z, \lim _{n \rightarrow \infty} S A x_{n}=S z$;

(13) conditionally reciprocal continuous [19] if $\mathscr{L}(A, S) \neq \emptyset$ implies that, for some $\left(x_{n}\right) \in \mathscr{L}(A, S)$, $\lim _{n \rightarrow \infty} A x_{n}=\lim _{n \rightarrow \infty} S x_{n}=z, \lim _{n \rightarrow \infty} A S x_{n}=$ $A z$, and $\lim _{n \rightarrow \infty} S A x_{n}=S z$;

(14) a $\mathscr{P}$-operator pair [10] if, for some $x \in C(A, S)$, $d(x, A x) \leq \operatorname{diam} C(A, S)$;

(15) a $\mathscr{J} \mathscr{H}$-operator pair [11] if, for some $x \in C(A, S)$, $d(x, A x) \leq \operatorname{diam} P C(A, S)$;
(16) a generalized $\mathscr{J} \mathscr{H}$-operator pair of order $n \in \mathbb{N}[12]$ if, for some $x \in C(A, S), d(x, A x) \leq(\operatorname{diam} P C(A, S))^{n}$;

(17) a $\mathscr{P} \mathscr{D}$-operator pair [14] if, for some $x \in C(A, S)$, $d(A S x, S A x) \leq \operatorname{diam} P C(A, S)$;

(18) $S$-biased [17] if, for all $\left(x_{n}\right) \in \mathscr{L}(A, S)$, $\alpha d\left(S x_{n}, S A x_{n}\right) \leq \alpha d\left(A x_{n}, A S x_{n}\right)$, where $\alpha=$ lim sup or $\alpha=\lim$ inf;

(19) weakly $S$-biased [17] if, for all $x \in X, x \in C(A, S)$ implies that $d(S A x, S x) \leq d(A S x, A x)$;

(20) occasionally weakly $S$-biased $[11,18]$ if, for some $x \in$ $C(A, S), d(S A x, S x) \leq d(A S x, A x)$

Note that the conditions (7), (8), and (9) are purely settheoretical and do not depend on the metric structure of $(X, d)$. All other conditions are metrical and could change if the metric of the space is changed (or some other structure of the space is applied).

In Table 1, we state which of these properties trivially hold (or can never hold) if one (or both) of the sets $C(A, S)$ and $\mathscr{L}(A, S)$ is empty or nonempty. Also, some of implications between these conditions obviously hold in some of these cases. Note that $\mathscr{L}(A, S)=\emptyset \Rightarrow C(A, S)=\emptyset$ (and hence, $C(A, S) \neq \emptyset \Rightarrow \mathscr{L}(A, S) \neq \emptyset)$.

Counter examples for some of the reverse implications are given in [8, Example in Section 2], [14, Example 3.1], [21, Example], and [24, Example 2.12].

The following are some other implications (mostly clear from definitions) that hold between the introduced notions. When it is not obvious that the reverse implication does not hold, a reference for a counterexample is given.

$(1) \Rightarrow(3)$. For the reverse implication see [3, Examples 2.1 and 2.2].

$(4) \Rightarrow(2)$. For the reverse implication see [25, Example 1].

$(3) \wedge(2) \Rightarrow(5)$ and $(6) \wedge(2) \Rightarrow(5)$. For the reverse implications see [26, Example 2.3].

$(3) \Rightarrow(6)$. For the reverse implication see [16, Example 1.2].

$(5) \Rightarrow(6)$ (for the reverse implication take arbitrary mappings satisfying $\mathscr{L}(A, S)=\emptyset)$ and $(5) \Rightarrow(2)$.

(2) and (7) are independent of each other (see [27, Examples 2.1-2.2]). 
$(3) \Rightarrow(7)$. For the reverse implication see, for example, [28, Example 2.3] (note that a part of the definition of the mapping $A$ in this example is missing; it should be, for example, $A x=(x+5) / 5$ for $5<x \leq 20)$.

(6) does not imply (7) (see [16, Example 1.3]).

(3) and (8) are independent (in one direction take any pair with $\mathscr{L}(A, S)=\emptyset$, and for the reverse see [9, Example 1.1]).

(4) and (8) are independent of each other (see [9, Examples 1.1-1.3]).

$(8) \Rightarrow(5)$. For the reverse implication see $[15$, Example $1.2]$.

$(8) \Rightarrow(6)$ and $(8) \Rightarrow(9)$. For the reverse implications take arbitrary mappings satisfying $\mathscr{L}(A, S)=\emptyset$ (and hence, $C(A, S)=\emptyset)$.

(9) does not imply (7) (see [9]).

$(3) \Rightarrow(10)$. For the reverse implication see $[16$, Example 1.4].

(4) and (10) are independent of each other. An example of a noncompatible pair which is not faintly compatible is [16, Example 1.5] (see also [9, Example 1.1]). Any pair of commuting that maps with $C(A, S) \neq \emptyset$ is faintly compatible and not noncompatible.

$(8) \Rightarrow(10)$. For the reverse implication see $[16$, Example 1.7] or [29, Example 2.1].

(11) and (12) are independent conditions, (in one direction, take arbitrary pair satisfying $\mathscr{L}(A, S)=\emptyset$, and in the other see [15, Example 1.4]).

$(11) \Rightarrow(13)$ and $(12) \Rightarrow(13)$. For the reverse implications see [19, Example 6].

$(7) \Rightarrow(14)$ and $(8) \Rightarrow(14)$. Indeed, it follows from (8) that there exists $x \in C(A, S)$ such that $A S x=S A x$; that is, $A x=S x \in C(A, S)$. But then $d(x, A x) \leq$ $\operatorname{diam} C(A, S)$.

(14) and (15) (together) do not imply either (7), or (8) (see [10, Example A]), or (17) (see [14, Example 3.3]).

$(15) \Rightarrow(16)$ (for the reverse implication and that (16) does not imply (8) see [12, Example 3.2]).

(17) does not imply (14), and (17) does not imply (15) (see [14, Example 3.4]).

$(3) \Rightarrow(18)$. For the reverse implication see [17].

$(18) \Rightarrow(19)$. For the reverse implication see [17].

$(7) \Rightarrow(19)$ and $(8) \Rightarrow(20)$. For the reverse implications see [30, Example 2.3] and [18, Example 3.2].

(19) does not imply (8) (see [30, Example 2.4]) and (20) does not imply (19) (see [18, Example 3.2]).

Finally, if $P C(A, S)$ is a singleton, then

$(8) \Rightarrow(7)[31$, Proposition 2.2],

$(5) \Rightarrow(7)[32$, Proposition 2.22], and

$(20) \Rightarrow(19)[24$, Proposition 2.11]

\section{Reducing Common Fixed Point Results to the Case of Weak Compatibility}

The following simple result can be used to show that several common fixed point theorems obtained recently are actually not generalizations of previously known results.

Proposition 2. Let $(X, d)$ be a metric space, and let $A, S$ : $X \rightarrow X$. Let the pair $(A, S)$ have exactly one point of coincidence; that is,

$$
P C(A, S)=\{w\} .
$$

Then conditions (7), (8), (9), and (17) are equivalent, and equivalent with the condition that the pair $(A, S)$ has a unique common fixed point.

Proof. Note first that $P C(A, S) \neq \emptyset$ implies that $C(A, S) \neq \emptyset$ and $\mathscr{L}(A, S) \neq \emptyset$ (just take $x_{n}=x$, where $x \in C(A, S)$, for all $n \in \mathbb{N}$ ) Consider the following.

$$
\begin{aligned}
& (7) \Rightarrow(8) \text { holds because } C(A, S) \neq \emptyset . \\
& (8) \Rightarrow(7) \text { follows by [31, Proposition 2.2]. } \\
& (8) \Leftrightarrow(9) \text { holds because } C(A, S) \neq \emptyset . \\
& (7) \Rightarrow(17) \text { was proved in }[14] \text {, and }(17) \Rightarrow(8) \text { follows } \\
& \text { because diam } P C(A, S)=\operatorname{diam}\{w\}=0 .
\end{aligned}
$$

In the case that $(A, S)$ has a unique POC, it was proved in [22] that condition (8) implies that $(A, S)$ has a unique common fixed point. The converse is obvious.

An easy example of mappings $A x=3 x-2$ and $S x=x^{2}$ on $X=[1,+\infty)$, when $P C(A, S)$ has two elements, shows that the condition that $P C(A, S)$ is a singleton cannot be removed from the previous proposition, neither does this proposition hold when $P C(A, S)=\emptyset$, as [19, Example 6] shows.

When considering two pairs of mappings, the following is a direct consequence of Proposition 2.

Corollary 3. Let $(A, S)$ and $(B, T)$ be two pairs of self-maps on a metric space $(X, d)$, satisfying

$$
P C(A, S)=P C(B, T)=\{w\} .
$$

Then the following conditions are equivalent.

(i) $(A, S)$ and $(B, T)$ both satisfy condition (7).

(ii) $(A, S)$ and $(B, T)$ both satisfy condition (8).

(iii) $(A, S)$ and $(B, T)$ both satisfy condition (17).

(iv) $A, B, S$, and $T$ have a unique common fixed point.

Applying Proposition 2 or Corollary 3, it is easy to show that a lot of the results of papers cited in the references are actually not generalizations of previously known ones.

As a sample, consider Theorems 2.1 and 2.2 of [16]. $P C(A, S)$ in these assertions is a singleton. Further, mappings $A$ and $S$ have a unique common fixed point. By Proposition 2, it follows that the pair $(A, S)$ is weakly compatible (condition (4)). Hence, using formally weaker assumption (10) does not produce a more general assertion. 
Similarly, in Theorems 2.1 and 2.2 and Corollaries 2.1-2.6 of [33], applying Corollary 3 , we get that the pairs $(f, S)$ and $(g, T)$ are weakly compatible.

In the same way, it can be concluded that the following results are actually not generalizations of previously known ones: [6, Theorems 1.4 and 1.5]; [8, Theorem 2.1]; [19, Theorems 1-3 of Section 2]; [11, Theorems 2.8-2.12]; [14, Theorems 4.1, 4.4, 4.6, 4.8, 4.10, 4.12, and 5.1]; [18, Theorems 4.1, 4.6, 5.6, 6.2; Corollaries 4.3, 4.4]; [22, Lemma 1; Theorems 1-5; Corollaries 1-5]; [30, Theorem 2.5; Corollary 2.7]; [34, Theorems 1-4 and Corollaries 1-3]; [35, Theorems 3.1, 3.4, and 3.6; Corollaries 3.9, 3.10, and 3.11]; [36, Theorems 2.2 and 2.6]; [37, Theorems 2.1-2.3 and 3.1-3.4; Corollary 2.1]; [38, Theorem 1.1]; [39, Theorems 2.1 and 2.3; Corollaries 2.2, 2.4 and 2.5]; [40, Theorems 3.1-3.3]; [41, Theorems 2.1 and 2.2; Corollaries 2.1-2.3]; [42, Theorems 3.1-3.3 and 4.1-4.3]; [43, Theorems 2.1 and 2.2]; [44, Theorem 3.2; Corollaries 3.1 and 3.2]; [45, Theorems 2.3]; [46, Theorem 11; Corollary 13]; [47, Theorems 2.2 and 2.3]; [48, Theorems 2.1-2.5]; [49, Theorems 2.1, 2.4, and 2.10]; [50, Theorems 4.1, 4.2, and 5.1-5.5].

A different kind of conclusions can be made for the results from $[28,51,52]$.

Again, as a sample, consider [52, Theorem 2.1], which (abbreviated) reads as follows.

Let $f$ and $g$ be two pseudoreciprocal continuous selfmappings of a complete metric space $(X, d)$ such that $f X \subseteq$ $g X$ and satisfying certain contractive condition. If the pair $(f, g)$ is conditionally sequential absorbing, then $f$ and $g$ have a unique common fixed point.

It can be reformulated as follows.

Under the previous conditions, the pair $(f, g)$ is weakly compatible (i.e., satisfies condition (7)).

Indeed, the proof of [52, Theorem 2.1] shows that $f$ and $g$ have a unique common fixed point. The contractive condition easily implies that they also have a unique POC (i.e., $P C(f, g)$ is a singleton). Then, Proposition 2 implies that $(f, g)$ is weakly compatible (and occasionally weakly compatible, of the type PD, conditionally commuting). Hence, weak compatibility is again a natural (and the weakest possible) assumption for this kind of results.

Similar conclusions can be made for the following results: [28, Corollary 2.1]; [51, Theorems 1-3]; [52, Theorems 2.2 and 2.3].

Several very interesting results were also obtained for multivalued mappings. We just note [53-55].

It is interesting that in the case of hybrid pairs of mappings (one single-valued and one multivalued) conclusions similar to those from this paper cannot be made. Namely, it was shown in [31, Example 2.5] that in this case Proposition 2 no longer holds. Hence, for example, results from the papers [5659] cannot be directly obtained from previously known ones.

\section{Conflict of Interests}

The authors declare that there is no conflict of interests regarding the publication of this paper.

\section{Acknowledgment}

The first and the second authors are grateful to the Ministry of Education, Science and Technological Development of Serbia. The third author acknowledges with thanks the Deanship of Scientific Research (DSR), King Abdulaziz University Jeddah, for financial support.

\section{References}

[1] G. Jungck, "Commuting mappings and fixed points," The American Mathematical Monthly, vol. 83, no. 4, pp. 261-263, 1976.

[2] S. Sessa, "On a weak commutativity condition of mappings in fixed point considerations," Publications de l'Institute Mathematique, vol. 32, no. 46, pp. 149-153, 1982.

[3] G. Jungck, "Compatible mappings and common fixed points," International Journal of Mathematics and Mathematical Sciences, vol. 9, no. 4, pp. 771-779, 1986.

[4] R. P. Pant, "Common fixed point theorems for contractive maps," Journal of Mathematical Analysis and Applications, vol. 226, no. 1, pp. 251-258, 1998.

[5] G. Jungck, "Common fixed points for noncontinuous nonself maps on nonmetric spaces," Far East Journal of Mathematical Sciences, vol. 4, no. 2, pp. 199-215, 1996.

[6] R. P. Pant and R. K. Bisht, "Occasionally weakly compatible mappings and fixed points," Bulletin of the Belgian Mathematical Society. Simon Stevin, vol. 19, no. 4, pp. 655-661, 2012.

[7] M. Aamri and D. El Moutawakil, "Some new common fixed point theorems under strict contractive conditions," Journal of Mathematical Analysis and Applications, vol. 270, no. 1, pp. 181188, 2002.

[8] M. A. Al-Thagafi and N. Shahzad, "Generalized $I$-nonexpansive selfmaps and invariant approximations," Acta Mathematica Sinica, vol. 24, no. 5, pp. 867-876, 2008.

[9] V. Pant and R. P. Pant, "Common fixed points of conditionally commuting maps," Fixed Point Theory, vol. 11, no. 1, pp. 113-118, 2010.

[10] H. K. Pathak and N. Hussain, "Common fixed points for $P$ operator pair with applications," Applied Mathematics and Computation, vol. 217, no. 7, pp. 3137-3143, 2010.

[11] N. Hussain, M. A. Khamsi, and A. Latif, "Common fixed points for $J H$-operators and occasionally weakly biased pairs under relaxed conditions," Nonlinear Analysis. Theory, Methods \& Applications, vol. 74, no. 6, pp. 2133-2140, 2011.

[12] W. Sintunavarat and P. Kumam, "Common fixed point theorems for generalized $\mathrm{JH}$-operator classes and invariant approximations," Journal of Inequalities and Applications, vol. 2011, article 67, 2011.

[13] R. P. Pant, "Common fixed points of Lipschitz type mapping pairs," Journal of Mathematical Analysis and Applications, vol. 240, no. 1, pp. 280-283, 1999.

[14] H. K. Pathak and Deepmala, "Common fixed point theorems for $P D$-operator pairs under relaxed conditions with applications," Journal of Computational and Applied Mathematics, vol. 239, pp. 103-113, 2013.

[15] H. Bouhadjera and C. Godet-Thobie, "Common fixed theorems for pairs ofsubcompatible maps," http://arxiv.org/abs/0906.3159.

[16] R. K. Bisht and N. Shahzad, "Faintly compatible mappings and common fixed points," Fixed Point Theory and Applications, vol. 2013, article 156, 2013. 
[17] G. Jungck and H. K. Pathak, "Fixed points via 'biased maps," Proceedings of the American Mathematical Society, vol. 123, no. 7, pp. 2049-2060, 1995.

[18] H. Bouhadjera and A. Djoudi, "Fixed point for occasionally weakly biased maps," Southeast Asian Bulletin of Mathematics, vol. 36, no. 4, pp. 489-500, 2012.

[19] R. P. Pant and R. K. Bisht, "Common fixed point theorems under a new continuity condition," Annali dell' Universita di Ferrara, vol. 58, no. 1, pp. 127-141, 2012.

[20] P. P. Murthy, "Important tools and possible applications of metric fixed point theory," Nonlinear Analysis, vol. 47, pp. 34793490, 2001.

[21] M. A. Al-Thagafi and N. Shahzad, "A note on occasionally weakly compatible maps," International Journal of Mathematical Analysis, vol. 3, no. 1-4, pp. 55-58, 2009.

[22] G. Jungck and B. E. Rhoades, "Fixed point theorems for occasionally weakly compatible mappings," Fixed Point Theory, vol. 7, no. 2, pp. 287-296, 2006.

[23] G. Jungck and B. E. Rhoades, "Erratum: Fixed point theorems for occasionally weakly compatible mappings," Fixed Point Theory, vol. 9, pp. 383-384, 2008, Fixed Point Theory, vol. 7, no. 2, pp. 287-296, 2006.

[24] M. A. Alghamdi, S. Radenović, and N. Shahzad, "On some generalizations of commuting mappings," Abstract and Applied Analysis, vol. 2012, Article ID 952052, 6 pages, 2012.

[25] J.-X. Fang and Y. Gao, "Common fixed point theorems under strict contractive conditions in Menger spaces," Nonlinear Analysis. Theory, Methods \& Applications, vol. 70, no. 1, pp. 184193, 2009.

[26] F. Rouzkard, M. Imdad, and H. K. Nashine, "New common fixed point theorems and invariant approximation in convex metric spaces," Bulletin of the Belgian Mathematical Society. Simon Stevin, vol. 19, no. 2, pp. 311-328, 2012.

[27] H. K. Pathak, R. Rodríguez-López, and R. K. Verma, “A common fixed point theorem using implicit relation and property (E.A) in metric spaces," Filomat, vol. 21, no. 2, pp. 211-234, 2007.

[28] D. Gopal, M. Imdad, and M. Abbas, "Metrical common fixed point theorems without completeness and closedness," Fixed Point Theory and Applications, vol. 2012, article 18, 2012.

[29] M. Imdad, J. Ali, and M. Tanveer, "Remarks on some recent metrical common fixed point theorems," Applied Mathematics Letters, vol. 24, no. 7, pp. 1165-1169, 2011.

[30] N. Hussain and H. K. Pathak, "Subweakly biased pairs and Jungck contractions with applications," Numerical Functional Analysis and Optimization, vol. 32, no. 10, pp. 1067-1082, 2011.

[31] D. Đorić, Z. Kadelburg, and S. Radenović, "A note on occasionally weakly compatible mappings and common fixed points," Fixed Point Theory, vol. 13, no. 2, pp. 475-479, 2012.

[32] M. Abbas, D. Gopal, and S. Radenović, "A note on recent introduced commutativeconditions," Indian Journal of Mathematics (IJM) Bulletin of the Allahabad Mathematical Society. In press.

[33] M. K. Jain, B. E. Rhoades, and A. S. Saluja, "Fixed point theorems for occasionally weakly compatible expansive mappings," Journal of Advanced Mathematical Studies, vol. 5, no. 2, pp. 54$58,2012$.

[34] M. Abbas and B. E. Rhoades, "Common fixed point theorems for occasionally weakly compatible mappings satisfying a generalized contractive condition," Mathematical Communications, vol. 13, no. 2, pp. 295-301, 2008.

[35] A. Aliouche and V. Popa, "Common fixed point theorems for occasionally weakly compatible mappings via implicit relations," Filomat, vol. 22, no. 2, pp. 99-107, 2008.
[36] G. V. R. Babu and G. N. Alemayehu, "Common fixed point theorems for occasionally weakly compatible maps satisfying property (E.A) using an inequality involving quadratic terms," Applied Mathematics Letters, vol. 24, no. 6, pp. 975-981, 2011.

[37] A. Bhatt, H. Chandra, and D. R. Sahu, "Common fixed point theorems for occasionally weakly compatible mappings under relaxed conditions," Nonlinear Analysis. Theory, Methods \& Applications, vol. 73, no. 1, pp. 176-182, 2010.

[38] R. K. Bisht and R. P. Pant, "A critical remark on 'Fixed point theorems for ocasionally weakly compatible mappings"' Journal of the Egyptian Mathematical Society, 2013.

[39] H. Bouhadjera, "On unique common fixed point theorems for three and four self mappings," Filomat, vol. 23, no. 3, pp. 115-123, 2009.

[40] H. Chandra and A. Bhatt, "Fixed point theorems for occasionally weakly compatible maps in probabilistic semi-metric space," International Journal of Mathematical Analysis, vol. 3, no. 9-12, pp. 563-570, 2009.

[41] S. Chauhan and B. D. Pant, "Common fixed point theorems for occasionally weakly compatible mappings using implicit relation," The Journal of the Indian Mathematical Society, vol. 77, no. 1-4, pp. 13-21, 2010.

[42] L. B. Ćirić, B. Samet, and C. Vetro, "Common fixed point theorems for families of occasionally weakly compatible mappings," Mathematical and Computer Modelling, vol. 53, no. 5-6, pp. 631636, 2011.

[43] N. Hussain, M. H. Shah, and S. Radenović, "Fixed points of weakly contractions through occasionally weak compatibility," Journal of Computational Analysis \& Applications, vol. 13, no. 3, pp. 532-543, 2012.

[44] M. Imdad, J. Ali, and V. Popa, "Impact of occasionally weakly compatible property on common fixed point theorems for expansive mappings," Filomat, vol. 25, no. 2, pp. 79-89, 2011.

[45] M. Imdad and A. H. Soliman, "Some common fixed point theorems for a pair of tangential mappings in symmetric spaces," Applied Mathematics Letters, vol. 23, no. 4, pp. 351-355, 2010.

[46] B. D. Pant and S. Chauhan, "Common fixed point theorem for occasionally weakly compatible mappings in Menger space," Surveys in Mathematics and its Applications, vol. 6, pp. 1-7, 2011.

[47] R. P. Pant and R. K. Bisht, "Common fixed point of pseudo compatible maps," Revista de la Real Academia de Ciencias, Fisicas y Naturales A, 2013.

[48] K. P. R. Sastry, G. A. Naidu, P. V. S. Prasad, V. Madhavi Latha, and S. S. A. Sastri, "A critical look at fixed point theorems for occasionally weakly compatible maps in probabilistic semimetric spaces," International Journal of Mathematical Analysis, vol. 4, no. 25-28, pp. 1341-1348, 2010.

[49] M. H. Shah, S. Simić, N. Hussain, A. Sretenović, and S. Radenović, "Common fixed points theorems for occasionally weakly compatible pairs on cone metric type spaces," Journal of Computational Analysis and Applications, vol. 14, no. 2, pp. 290-297, 2012.

[50] Deepmala and H. K. Pathak, "Some common fixed point theorems for D-operator pair with applications to nonlinear integral equations," Nonlinear Functional Analysis \& Applications, vol. 18, pp. 205-218, 2013.

[51] R. P. Pant, R. K. Bisht, and D. Arora, "Weak reciprocal continuity and fixed point theorems," Annali dell'Universitá di Ferrara. Sezione VII. Scienze Matematiche, vol. 57, no. 1, pp. 181-190, 2011.

[52] D. K. Patel, P. Kumam, and D. Gopal, "Some discussion on the existence of common fixed points for a pair of maps," Fixed Point Theory and Applications, vol. 2013, article 187, 2013. 
[53] W.-Sh. Du, E. Karapinar, and N. Shahzad, "The study of fixed point theory for various multivalued non-self maps," Abstract \& Applied Analysis, vol. 2013, Article ID 938724, 9 pages, 2013.

[54] F. Khojasteh and V. Rakočević, "Some new common fixed point results for generalized contractive multi-valued non-selfmappings," Applied Mathematics Letters, vol. 25, no. 3, pp. 287293, 2012.

[55] B. D. Rouhani and S. Moradi, "Fixed point of multi-valued generalized $\Phi$-weak contractive mapping," Fixed Point Theory \& Applications, vol. 2010, Article ID 708984, 2010.

[56] M. Abbas and B. E. Rhoades, "Common fixed point theorems for hybrid pairs of occasionally weakly compatible mappings satisfying generalized contractive condition of integral type," Fixed Point Theory and Applications, vol. 2007, Article ID 54101, 9 pages, 2007.

[57] M. Abbas and B. E. Rhoades, "Common fixed point theorems for hybrid pairs of occasionally weakly compatible mappings satisfying generalized contractive condition of integral type," Fixed Point Theory and Applications, vol. 2008, Article ID 274793, 2 pages, 2008.

[58] A. Aliouche and V. Popa, "General common fixed point theorems for occasionally weakly compatible hybrid mappings and applications," Novi Sad Journal of Mathematics, vol. 39, no. 1, pp. 89-109, 2009.

[59] S. Chauhan and P. Kumam, "Common fixed point theorem for occasionally weakly compatible mappings in probabilistic metric spaces," Thai Journal of Mathematics, vol. 11, no. 2, pp. 285292, 2013. 


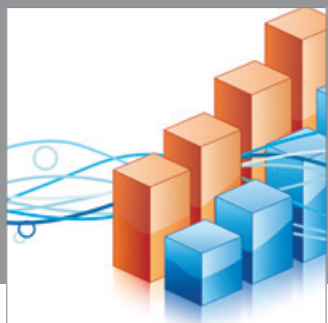

Advances in

Operations Research

mansans

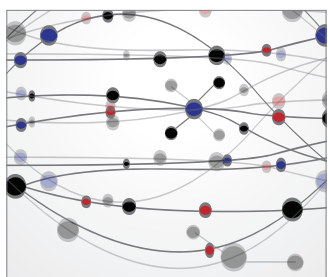

The Scientific World Journal
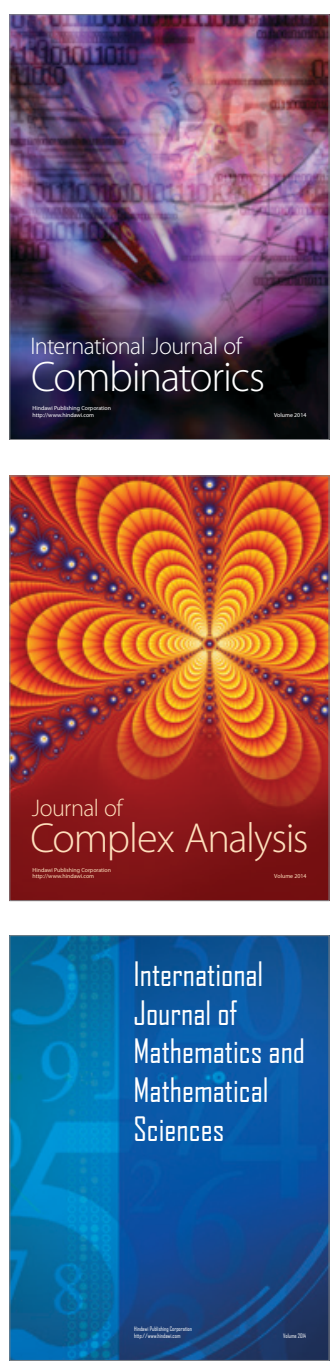
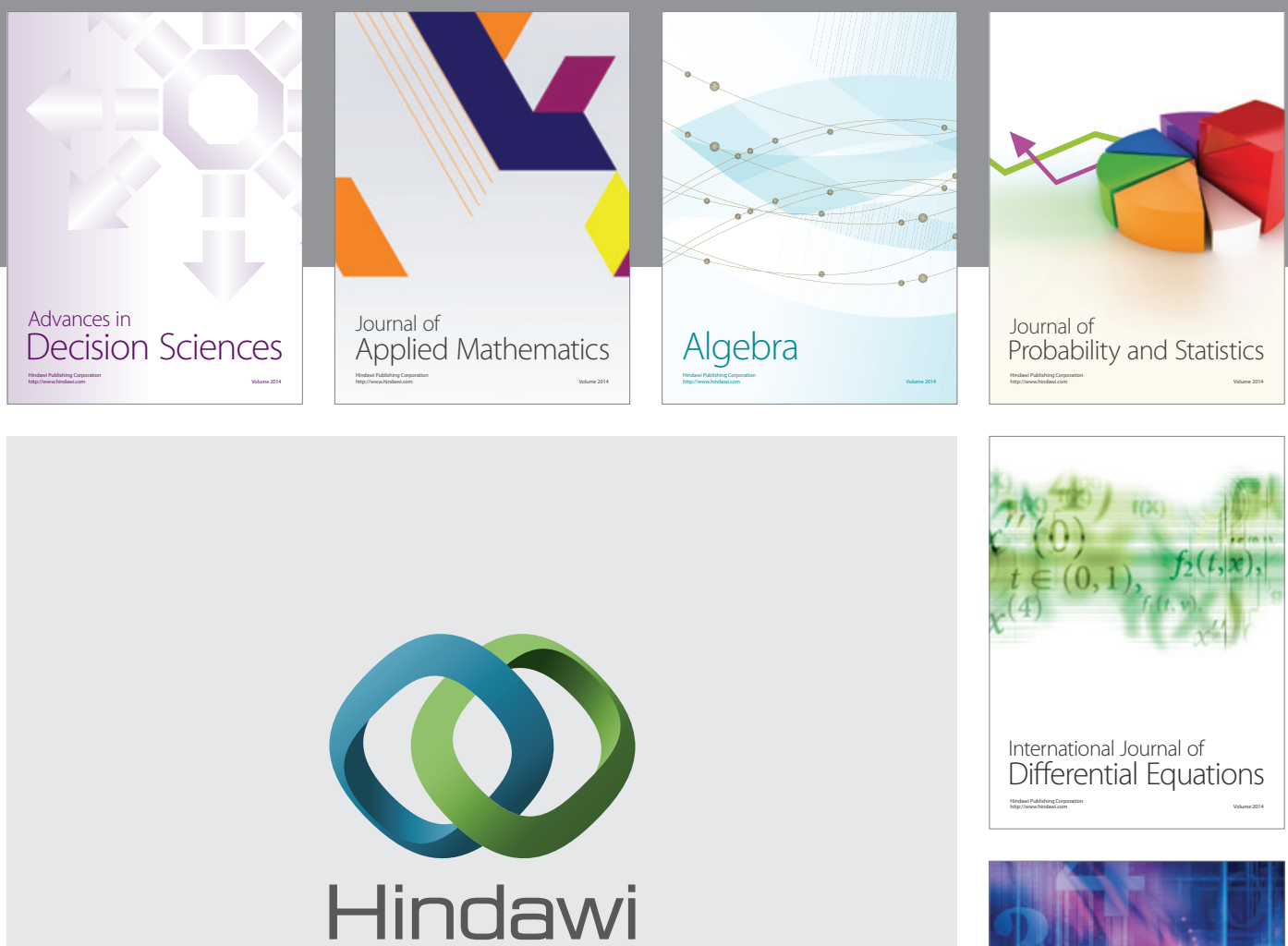

Submit your manuscripts at http://www.hindawi.com
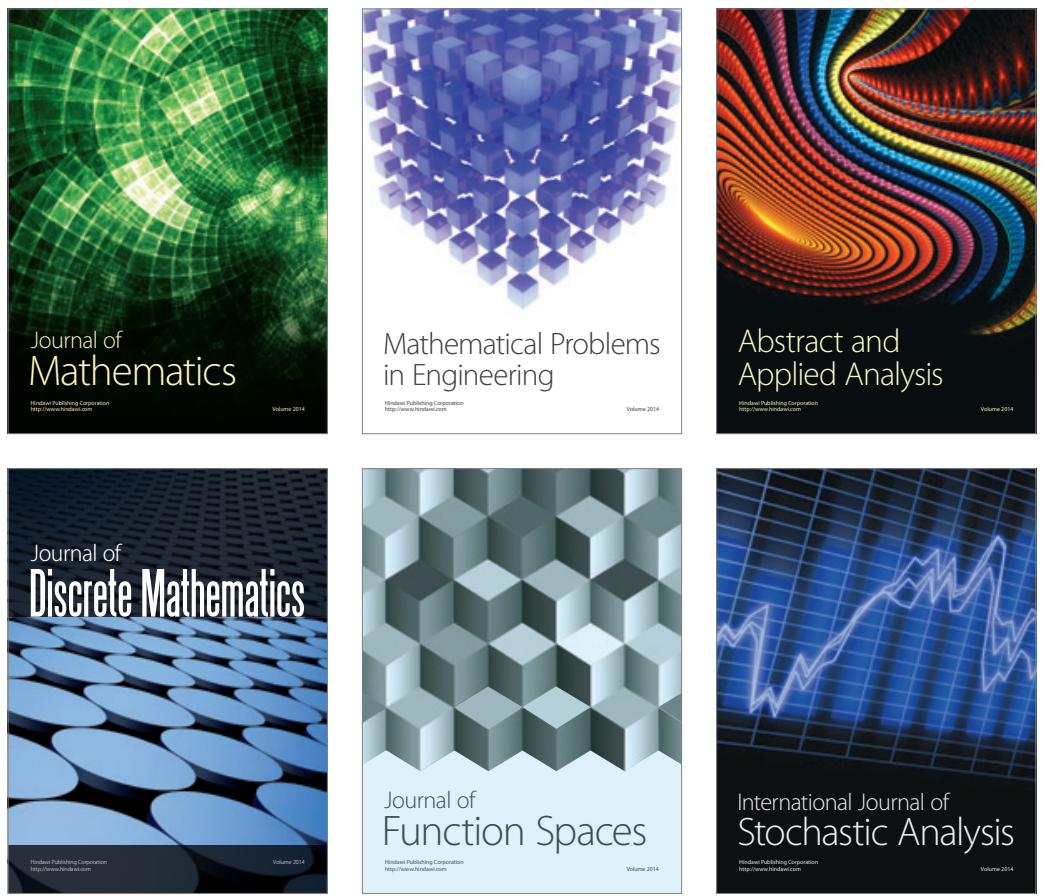

Journal of

Function Spaces

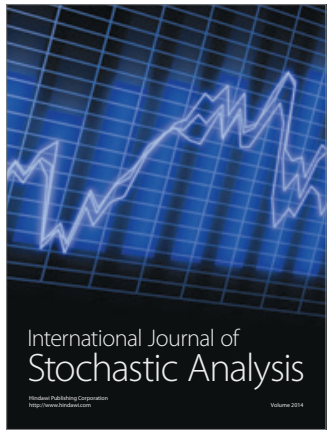

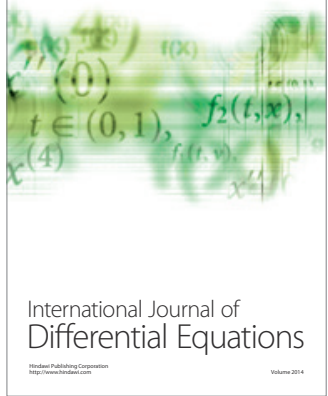
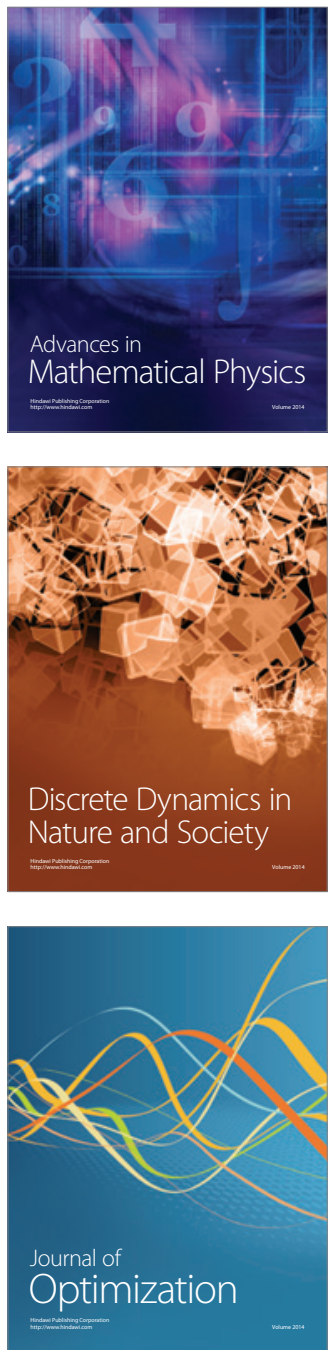